\title{
APROXIMACIÓN AL CONCEPTO DE RACIONALIDAD JURÍDICA EN NIKLAS LUHMANN ${ }^{1}$
}

Legal Approach to the Concept of Rationality in Niklas Luhmann ${ }^{1}$

Juan David Castro Villadiego ${ }^{2}$ Felipe David González Palma ${ }^{3}$ Alejandro Raúl Sarmiento Cantillo ${ }^{4}$

Fecha de Recepción: Noviembre 3 de 2014

Fecha de Aceptación: Noviembre 10 de 2014

SUMARIO: 1. Introducción; 2. Metodología; 3. Resultados; 3.1. La racionalidad del derecho en Niklas Luhmann; 3.2. El derecho y la realidad por fuera del derecho; 3.3. La autopoiesis del sistema jurídico y la colonización de la subjetividad del sujeto; 3.4 Los errores comunicativos del sistema;

4. Conclusiones; 5. Referencias bibliográficas.

\footnotetext{
${ }^{1}$ El presente trabajo es un informe parcial de investigación del proyecto Manual de teoría de la interpretación en el derecho. Los autores de este informe actuaron como COINVESTIGADORES del proyecto, financiado por la Universidad de Cartagena; iniciado en el año 2011 y culminado en 2012.

${ }^{2}$ Abogado de la Facultad de Derecho y Ciencias Políticas de la Universidad de Cartagena. Investigador perteneciente al grupo de investigación Conflicto y Sociedad. Correo electrónico:lautmull@gmail.com

${ }^{3}$ Egresado de la Facultad de Derecho y Ciencias Políticas de la Universidad de Cartagena. Correo electrónico: felipedgp@ hotmail.com

${ }^{4}$ Egresado de la Facultad de Derecho y Ciencias Políticas de la Universidad de Cartagena. Correo electrónico: arsc27@outlook.com
} 


\title{
COMO SE CITA ESTE ARTÍCULO (APA 6)
}

Castro Villadiego, J. D., González Palma, F. D., Sarmiento Cantillo, A. R. (2014) Aproximación al concepto de racionalidad jurídica en Niklas Luhmann. (Y. Carrillo De la rosa, Ed.) Revista Jurídica Mario Alario D'Filippo, VII (13), pág. 102-113

\section{RESUMEN:}

En este artículo de investigación se estudian las implicaciones de la teoría constructivista, en referencia al concepto de racionalidad jurídica en Niklas Luhmann; se parte de las consideraciones realizadas por Niklas Luhmann en la teoría de los sistemas autopoiéticos, con la disposición de difundir una posición crítica frente a las implicaciones que se crean al momento de aplicar los concepto Luhmannianos en la racionalidad jurídica.

\begin{abstract}
In this research paper the implications of constructivist theory are studied, referring to the concept of legal rationality in Niklas Luhmann; It is part of the considerations made by Niklas Luhmann's theory of autopoietic systems, with the provision of spreading a critical position on the implications that are created when applying the concept Luhmannianos legal rationality.
\end{abstract}

\section{PALABRAS CLAVE}

Constructivismo, autopoiesis, racionalidad jurídica, justicia, sistemas.

\section{KEYWORDS}

Constructivism, autopoiesis, legal rationality, justice, systems. 


\section{INTRODUCCIÓN}

Es frecuente ver en la práctica judicial como muchas veces sin la necesidad de adentrarse por completo en la particularidad de cada caso, los fallos de los jueces nos dejan con un sin sabor, con una herida que brota inconformidad, con una a veces leve o a veces fuerte sensación que se expresa en un "si yo hubiese sido el juez otra hubiese sido la decisión", o que nos lleva a dar de bruces con esa tan particular conclusión tan común en quienes nos adentramos en el estudio del derecho que se reduce a "el derecho es una mierda". Son todas esas sensaciones producto de una estructura de valores y creencias que tenemos cada uno como sujeto inserto en una realidad social de la que somos en alguna medida conscientes, que vemos a través de una serie de preconcepciones, adquiridas a lo largo de ese periodo de constante formación que es la vida consciente del ser humano dentro de una sociedad que reacciona frente al derecho.

La inconformidad del sujeto como ser humano -y no como persona- con el derecho es en parte producto de la insatisfacción de una expectativa de justicia, expectativa coartada de antemano por la naturaleza de ciertas normas y figuras jurídicas que sacrifican criterios de libertad, en pos de criterios de orden público o interés general. Pero cuando las propias figuras jurídicas llamadas a la realización de lo materialmente justo, ven limitado su ámbito de protección a la falta de una firma, un sello, media hora, un minuto, un centavo, etc., llegando a tal punto que lo que el proceso judicial tiene como cierto, está en completa desconexión a lo que los seres humanos tenemos y podemos percibir como real; lo que denota de forma clara, la manifiesta diferencia en el proceso cognitivo del derecho y el del ser humano en cuanto a humano. Esto no podría ser de otra manera, el derecho no tiene sentidos (desde el punto de vista biológico), no puede crearse una realidad como lo hacen los seres humanos individualmente considerados, no puede acceder a los fenómenos de la forma como lo hacen los seres humanos; $y$ al no poder hacerlo, tiene vedado acceder al mundo sensible, quedándole solo una alternativa, acceder a ese mundo sensible a través del lenguaje.

Sin embargo, el acceso del derecho al mundo o a la sociedad propiamente dicha, no se da solo a través del lenguaje; sino que se vale de la comunicación, de la que el lenguaje es presupuesto; el derecho solo conoce lo que los seres humanos perciben y comunican, a través del lenguaje. Es así como la teoría discursiva de Habermas reconoce que el derecho es el resultado del proceso comunicativo o intersubjetivo; la intersubjetividad asume el papel estelar en la construcción de la verdad jurídica o la verdad para el derecho; logrando así, explicar en parte el proceso cognitivo del derecho "La intersubjetividad toma el puesto del sujeto epistémico kantiano. Es el auténtico consenso de la comunidad comunicativa y no la conciencia del individuo autónomo el que determina la verdad en cuestiones cognitivas y normativas". (Teubner, 2002)

Parafraseando a Teubner, hemos de decir que la teoría de Habermas se pierde en la autoreferencialidad del discurso que se legitima a sí mismo; según Teubner, Habermas intenta evadir la "paradoja de la autoreferencialidad" echando mano de soluciones que para aquel resultan trascendentalistas, y que llevan a que "el sujeto epistémico de Habermas oscile entre dos campos". (Teubner, 2002)

Ante la deficiencia de la teoría discursiva de Habermas, la incapacidad de superar las paradojas de la 
autoreferencialidad; la teoría de la autopoiesis de Niklas Luhmann parece poder explicar el derecho a partir de su autoreferencialidad, hablando de los discursos que fundamentan las instituciones en el derecho como "sistemas autopoiéticos"; "los discursos solo se justifican en su circularidad y solo pueden producir regulaciones que se regulan así mismas, que gobiernan la transformación de sus propias regularidades" (Teubner, 2002); en palabras del propio Luhmann "el derecho mismo es quien produce todas las distinciones y descripciones que utiliza, y que la unidad del derecho no es más que el hecho de su autoproducción: "autopoiesis"'. (Luhmann, 2005)

Luhmann describe el derecho como "un sistema social autopoiético, clausurado en su operación" (Luhmann, 2005), haciendo del derecho un sistema independiente de los sujetos, diferenciándolo de la sociedad misma - el derecho es para Luhmann un subsistema al interior de la sociedad-.

De una manera parecida a la de Habermas, Luhmann considera que el sistema jurídico es pura comunicación. La elaboración constante que hace el derecho de sí mismo se produce en las comunicaciones; la cuales provienen de otras comunicaciones originadas en el sistema jurídico ${ }^{5}$.

Por fuera del derecho, también hay otros mundos o sistemas creados desde la realidad sistémica, un ejemplo de este mundo sistémico sería el biológico, en el que participan seres humanos de carne y hueso, pero solo en cuanto a seres vivientes.

Para Luhmann el derecho es un discurso social desindividualizado, una realidad construida que no tiene acceso a otras realidades. Teubner, refiriéndose a las personas consideradas por el derecho, afirma "con las que trata el derecho no son las personas de carne y hueso, no son lo seres humanos dotados de una mente y un cerebro... Son meros constructos, artefactos semánticos producidos por el discurso jurídico" (Teubner, 2002); los seres humanos en cuanto seres comunicativos son utilizados por el derecho para obtener de ellos las comunicaciones que lo crean.

\section{METOdología}

La investigación es cualitativa porque estudiaremos aspectos propios de la realidad jurídica, intentando a través de la caracterización de la misma, determinar cómo se aproxima la realidad jurídica al individuo, y como se relaciona el derecho con el jurista.

La investigación es descriptiva porque se busca la descripción del fenómeno. Describir las diferentes características que le son propias a la realidad jurídica y su proceso de creación.

La investigación es Bibliográfica porque conforme a lo anterior, se busca localizar, evaluar y clasificar el material doctrinario desarrollado alrededor del problema de investigación.

El objeto de esta investigación toma en consideración aspectos relacionados con esas regulaciones

\footnotetext{
${ }^{5}$ Los sistemas psíquicos observan el derecho (y no lo producen), de otro modo el derecho quedaría encerrado en la profundidad de aquello que Hegel alguna vez expresó: "en la oscura interioridad de los pensamientos". Véase (Luhmann, 2005)
} 
que tienen el carácter de jurídicas, que configuran el mundo de lo juicio, por lo que la presente es una investigación jurídica. Todo método científico se determina en función del objeto al cual se aplica: la observación y la experimentación se pueden, por ejemplo, aplicar en las ciencias naturales como medios de aproximación al estudio de los fenómenos de que estas se ocupan, pero no son utilizables en el análisis de fenómenos producidos por la acción del hombre, como lo es el derecho. El objeto del derecho en la investigación jurídica está constituido por fuentes formales (Giraldo, 1985), las que por una parte se explicitan a través del lenguaje, elemento de naturaleza simbólica y por otra, constituyen postulados de deber ser. La aproximación al derecho como objeto de conocimiento debe orientarse, por consiguiente, a la aprehensión del contenido significativo de los términos que integran cada una de las fuentes, y a la determinación del alcance de estas de acuerdo con el fin para el que fueron expedidas. Dicho objeto de investigación, enfocando desde el objetivo general y único ${ }^{6}$ de esta investigación que se circunscribe a la determinación del por qué la realidad jurídica en sí no es un fenómeno que surge del mundo hacia los individuos recogidos por las normas, sino que dichos individuos intervienen en el proceso de creación del derecho a través de su lenguaje amerita un especial tratamiento, toda vez que no se trata aquí del análisis de manifestaciones del derecho como normas u ordenamiento jurídico en general, sino sobre la realidad jurídica que generan.

\section{RESULTADOS}

Los resultados presentados es producto de la investigación adelantada por los autores. Procederemos en principio a dilucidar respecto al tópico verdad en el derecho dentro de la teoría consensual y la teoría constructivista; de lo que se siguen una serie de consideraciones críticas en torno a las implicaciones de las tratadas teorías, que alcanzan planos que trascienden al derecho mismo y obligan a remisiones de formas de aproximación del derecho a la realidad o como la realidad interactúa con el derecho.

\subsection{LA RACIONALIDAD DEL DERECHO EN NIKLAS LUHMANN}

La epistemología constructivista del derecho es explicativa respecto a las consideraciones de la verdad en el derecho; al aceptar que la realidad del derecho es una realidad construida por el sujeto, se hacen innecesarias las elucubraciones relativas a una posible aplicación de la teoría de la verdad como correspondencia Ontológica-semántica-formal en el campo del derecho; sin embargo en alguna medida asiste razón al profesor Apel cuando afirma que "La teoría de la verdad como correspondencia contiene una intuición natural fundamental que se presupone en todas las teorías posibles de la verdad" (Apel,1991). Es imposible establecer una relación sujeto-objeto; relación imprescindible para la teoría de la correspondencia, ya que de ella se extraen los elementos para la realización del juicio de correspondencia.

El proceso racional que utiliza la teoría de la correspondencia inicia desde el sujeto cognoscente,

\footnotetext{
${ }^{6}$ No se plantean objetivos específicos por tratarse de una investigación de teoría jurídica pura basada en reflexiones de filosofía del derecho y ciencia de base cultural, tal como lo entiende Max Weber.
} 
quien reconstruye el objeto; es por eso que hablar de aplicación de una teoría de la correspondencia, supone que los juicios de correspondencia hechos por el sujeto son verificados por un observador externo; quien ve el proceso cognitivo como una relación objeto-objeto. iLa autorreferencialidad está por todas partes!

Es posible que el sistema jurídico coincida con representaciones ajenas a él; esto por lo general tienen ocurrencia cuando las representaciones de los individuos son iguales a las representaciones creadas por el derecho respecto a un mismo fenómeno - para tal caso citamos a modo de ejemplo el gran número de coincidencias de derecho y moral, en el evento de que existan reglas del sistema jurídico coherentes con las representaciones morales de los individuos-; en estos casos el origen de tal coincidencia oscila en una delgada línea entre la causalidad y la casualidad, ya que si bien el derecho en la tradición de occidente tiene un fuerte contenido moral -como admitieron gran cantidad de autores una vez superado el paradigma kelseniano-, no existe una relación necesaria entre la racionalidad del sistema jurídico y las consideraciones morales de las personas. El individuo no es un sujeto epistémico en el sistema de derecho; ni el derecho accede a las representaciones del individuo, sino que instrumentaliza sus comunicaciones.

Recapitulando lo escrito hasta aquí, es preciso fijar estas conclusiones: a) La teoría de correspondencia no es suficiente para explicar la racionalidad en el sistema de derecho. Esta aunque pretenda explicar el derecho desde un análisis racional que vincula la norma y al intérprete; es incapaz de explicar la validez del acto de interpretación sin recurrir a una segunda observación; b) la autorreferencia es una trampa de la que de la que no puede eludir una teoría de la correspondencia; su intento de explicar el derecho a partir de una relación simple sujeto-objeto es insuficiente; c) la epistemología constructivista del derecho convierte la realidad del derecho en una realidad construida por el sujeto Derecho.

\subsection{EL DERECHOY LA REALIDAD POR FUERA DEL DERECHO}

La relación individuo-derecho en la teoría de Niklas Luhmann es compleja; decíamos en el párrafo anterior que un individuo no hace parte del sistema derecho, porque el derecho no está formado de individuos en cuanto ente carnal; el derecho al igual que la sociedad está formada de comunicaciones; los individuos pueden hacer parte de otro sistema que Luhmann llama biológico. Estos sistemas no se relacionan, funcionan construyendo fronteras. Se formula entonces la pregunta ¿Cómo es que no puede el derecho acceder al proceso psíquico del individuo?, si suponemos que el derecho dicta las normas de conducta de los individuos. También podemos interrogar ¿Dónde surge el derecho si el individuo no reconoce su contenido o desconoce su contenido? El profesor alemán responde a estas preguntas de la siguiente manera:

El reconocimiento no significa asentimiento por convicción; en una sociedad compleja el sistema solo puede funcionar si también funciona el sometido al derecho, si le es posible aprender libre de interferencias y si encaja en el sistema; el individuo sometido al derecho es una parte del sistema total, de tal modo que en último término es el sistema mismo el que produce el reconocimiento. (Kauffmann, 1992) 
Luhmann nos lleva por el camino que separar el proceso cognitivo del individuo del proceso cognitivo del derecho, y el proceso cognitivo del individuo mismo. La Teoría de la autopoiesis al suprimir al individuo pensante del proceso de elaboración del derecho se blinda contra la posibilidad de que la autoreferencialidad le explote en la cara al nuevo sujeto epistémico. Al señalar las comunicaciones como productoras y productos del sistema se hace sabiendo también que ellas pueden negar el sistema pero no autodestruirlo, ya que ellas son el presupuesto de funcionamiento del sistema, y no los destinatarios de la función del sistema:

"la comunicación, para ser verdaderamente autopoiética, debe contener en sí misma la posibilidad de su negación. Es por ello que la autopoiesis continúa su curso con independencia de que se produzcan (o no) efectos en el interior de los sistemas psíquicos. La continuación de la comunicación (autopoiética) solo puede determinarse por la propia comunicación". (Gómez-Jara, 2008)

Al hablar entonces de Derecho como comunicación; comunicaciones que produce el derecho y que expresa a través de normas, como una decisión judicial que aplica la norma al caso; en base a una interpretación de la realidad de la que el caso y la norma hacen parte; es una configurada relación objeto-objeto, el proceso de creación del derecho, la autopoiesis del derecho completa su primer ciclo en la sustantivación de la visión del juez expresada en la decisión.

El Derecho a través de la autopoiesis determina su realidad; la autopoiesis funciona a través de la clausura del sistema; queriendo siempre someter, ordenar, castrar la voluntad política transformista, ser realidad de realidades, el derecho pretende que la realidad sea una realidad jurídica legitimada por sí mismo.

En su afán logra que el sujeto individual deja de verse como ser humano y comienza a verse como el "constructo semántico"; a quien el derecho llama "persona". El derecho logra un efecto "Matrix"; los seres humanos que habitamos la "Matrix" somos autómatas llamados personas; jamás podremos acceder más que al mundo creado mediante el derecho, y la única forma de desconectarse de la "Matrix" es pensar por fuera de la ella.

Todo juicio de valor que se haga referente al derecho no afecta al sistema de derecho, porque per se es un juicio que está por fuera del sistema de racionalidad del derecho o puede ser una consideración dentro del sistema de derecho. El criterio de verdad del derecho es un criterio violento. Cuando hablamos aquí de violencia no debemos asimilar está a la fuerza o a la coerción. Bien podría decirse que el estado posee la fuerza que despliega sobre la población, y que regularmente manifiesta a través de coerción, estableciendo soluciones violentas a los problemas de la vida en sociedad donde es fácil ver el vector que sigue la fuerza que despliega el estado. Pero esa fuerza del estado ha encontrado nuevas formas de manifestación, y valiéndose de la cultura es como el Estado logra una aplicación no coercitiva de la fuerza con iguales efectos violentos.

"Ahora bien, 'fuerza' significa aquí no solamente la nuda coerción que identificamos de manera usual y algo ingenua con el uso de las armas, aunque sin duda este momento es originario, sino también la no menos importante fuerza imaginaria y simbólica que consiste en la determinación de unos caracteres o atributos que se establecen como esenciales (la 'unidad 
de cultura': lengua, religión, territorio y etnicidad) y de las formas - también esencializadascomo han de resolverse los conflictos." (Guardiola, 2004)

Cuando la fuerza no se impone simbólicamente, se usa la coerción para imponer racionalidad en el derecho; la acción racional del derecho puede ser observada en las actuaciones de los sujetos del sistema que crean el derecho; un juez o un alcalde tienen la capacidad declarar la verdad o la razón en el derecho, aunque la providencia o el acto administrativo no sean verdades, el sistema autopoiético las convierte en verdad. (Pagenestecher: 1905 citado por Neumann:2006 p.61)

La racionalidad del derecho no es deducible de un axioma de justicia o derecho material; la justicia material de acuerdo a la filosofía práctica kantiana solo será posible en los casos en que sus postulados coincidan con la justicia procedimental que impone el derecho como sistema autopoiético -es probable que sean coincidentes-, la justicia en el sistema derecho es casual; la justicia procedimental hace posible la existencia autopoiética del sistema ${ }^{7}$. Cualquier cuestionamiento que hagamos del derecho sobre un razones a nivel epistemológico de sus mandatos -ya sea en forma de reglas universales y abstractas o en normas individualizadas- será respondido con un contundente e inobjetable, i porque al derecho le da la gana!

\subsection{LA AUTOPOIESIS DEL SISTEMA JURÍDICO Y LA COLONIZACIÓN DE LA SUBJETIVIDAD DELINDIVIDUO ${ }^{8}$}

Con la teoría Luhmaniana, los seres humanos dejan de ser el centro de las organizaciones sociales; para ser remplazados por comunicaciones. Superando el realismo epistemológico y accediendo a un momento ulterior gobernado por el constructivismo epistemológico. El individuo y su subjetividad son remplazados por constructos lingüísticos, conceptos semánticos y por la subjetividad de la organización social. Auto-reflexión y auto-organización son el eje del sistema; atrás quedan las acciones intencionales de los seres humanos que configuraban los elementos esenciales de la sociedad. Los elementos básicos del sistema no es el sujeto Juan, ni las normas, ni legisladores. Los elementos ahora son las comunicaciones jurídicas. Esto equivale a decir:

"No son los seres humanos los que por medio de sus acciones intencionales producen el derecho como un artefacto cultural, sino que este como proceso comunicativo el que crea actores humanos como artefactos semánticos a través de sus operaciones jurídicas." (Teubner, 2002)

Este giro epistemológico es un rechazo a la intencionalidad -cualidad inmanente- del sujeto al interior del sistema. Como consecuencia el "poder" comunicativo del discurso social autónomo que llamamos derecho, absorbe al sujeto. El derecho como sistema se presenta ciego e indiferente de la intencionalidad del individuo.

\footnotetext{
${ }^{7}$ Utilizar la palabra justicia para el funcionamiento del sistema es un eufemismo; su uso podría ser paradójico. Si tenemos en consideración que la justicia procedimental o basada en criterios de corrección distributiva podría poner el derecho de frente a una justicia no tan justa.

${ }^{8}$ Cuando hablamos del sujeto nos referimos al ser humano considerado en su parte volitiva y no como un mero constructo de un sistema. En ninguna medida nos referimos al derecho como sujeto epistémico cuando hablamos de "sujeto".
} 
El derecho y los sistemas sociales son inmunes a la realidad "real"; encerrado en su realidad creada y reproducida autpoiéticamente; para de esta forma auto-validarse y justificarse, porque un reproche o cuestionamiento interno en el sistema a través de un metalenguaje implicaría su inevitable destrucción. No hay, ni puede haber, un punto de vista externo al derecho cuando hablamos de derecho, no podemos hablar de él por fuera de la realidad y esta la crea él mismo" (Mercado Pérez, 2008). Entonces, "el individuo sometido al derecho es una parte del sistema total, de tal modo que en último término es el sistema mismo el que produce el reconocimiento". (Kauffmann, 1992)

La criatura ha absorbido a su creador, le impone las reglas para su reconocimiento interior. El individuo cree ingenuamente hablar un lenguaje al interior del derecho; pero su comunicación es otro lenguaje -creado al interior del sistema-que habla por él; son "comunicaciones jurídicas". El sujeto es sometido por el derecho, su subjetividad remplazada por la presumible objetividad creada por el sistema mismo a partir de las comunicaciones internas. Esta es la colonización a la que hacemos referencia; consiste en intentar acortar los límites de su desarrollo individual.

La autopoiesis es una relación de sustantivación objetivante al interior del derecho; es el referente y esencia mediante el cual la autorreferencia en el sistema derecho es posible. La sustantivación objetivante es un proceso en el que se crean constructos lingüísticos o comunicativos al interior del sistema que finaliza en la objetivación o inserción a la vida del derecho de un objeto creado por el sistema. Como ejemplo podemos tomar los conceptos de antijuricidad, culpabilidad, principio de prohibición de regreso etc. El derecho fábrica su realidad, y a su vez este proceso indefinidamente repetido contribuye a que exista el derecho. (Mercado Pérez: 2008)

Este proceso utiliza subjetividades del sistema que sometidos a procedimientos de objetivización pasan a un sistema como reales dentro del sistema; la intencionalidad de los seres humanos no es tenida en cuenta en el proceso de creación de la verdad al interior del derecho. El individuo ha sido desterrado del sistema. El derecho es quien define su contenido y su estructura. Las comunicaciones del sistema son las creadoras del sujeto.

La colonización totalizadora que el derecho ejerce sobre el sujeto es infructuosa parcialmente; es imposible desde un punto de vista externo al sistema afectar la psiquis del sujeto; pese a ello la afectación al sujeto es notable. Al respecto nos dice Elías ${ }^{9}$.

"Cada vez más los hombre viven en un mundo de símbolos que ellos mismos han elaborado, y sin hacerse absoluta, la autonomía de sus enclaves se vuelve enorme."

Nos referimos al hecho de que la autonomía en los enclaves sociales humanos puede crecer, aunque ello sea relativo, nos ayudará tal vez a oponemos a un pensamiento inveterado y equívoco que viene imponiéndose entre nosotros. Pensamos y hablamos, en efecto, en conceptos que suscitan la impresión de que "sociedad» y «naturaleza», "objeto»

\footnotetext{
${ }^{9}$ En este caso para los que nos concierne entenderemos que cuando Elías se refiere a tiempo, es aplicable al Derecho y es como lo entendemos.
} 
$y$ "sujeto» existieran como entidades independientes entre sí. Se trata de un error que, sin una perspectiva de largo plazo, es difícil de combatir." (Norbert Elías, 1989)

El discurso se presenta con apariencia de objetividad, certeza y necesidad para el sujeto; condicionando el actuar de este. Pero al interior del sistema esas cualidades no son aparentes, sino que constituyen su propia realidad y el objeto mismo que permite su coherencia interna. Las costumbres lingüísticas nos llaman al engaño al decir de Norbert Elías, sobrevalorando en este caso la realidad "mítica" del sistema jurídico.

El sujeto es sometido a un conflicto permanente: ser colonizado por el derecho. Esto la desaparición de su determinación como sujeto. Pero a su vez se enfrenta a una disyuntiva poco percibida entre: Los hechos "reales" de la realidad externa, y los hechos "reales" de la realidad jurídica. Una disyuntiva esencial que al decir de Elías

"Cuando se traduce el sustantivo «tiempo" con su forma verbal y se estudia el problema de la determinación del tiempo, se da uno cuenta enseguida que no se pueden separar por completo las determinaciones de los sucesos sociales y de los hechos físicos." (Norbert Elías, 1989)

\subsection{LOS ERRORES COMUNICATIVOS EN EL SISTEMA}

Los presupuestos de la situación ideal de discurso son para Habermas el a priori de la intersubjetividad como sujeto epistémico; si ellos no se cumplen, no puede funcionar la comunicación; cuando no funciona la comunicación el derecho no podrá funcionar. Alexy (2003) analiza que el contenido sustancial de la justicia es consecuencia de la "Idealización", considerando esto y lo anteriormente planteado cabe aquí hacer el siguiente interrogante ¿Qué pasa si la "Idealización" es solo aparente o inexistente? La respuesta a esta pregunta no está muy lejos de lo que sucede en los mal llamados países tercermundistas, en cuanto al funcionamiento del sistema jurídico, ya que las diferentes problemáticas sociales, económicas y políticas que aquejan a dichos países, impiden que la totalidad de los actores de esas sociedades puedan participar de una verdadera dinámica deliberativa y democrática, al no darse los presupuestos de la situación de discurso ideal, lo cual podría decirse es generado por un masificado incumplimiento de las normas conformantes del ordenamiento jurídico; las pretensiones de certeza, validez e inteligibilidad son irrealizables, a pesar de los presupuestos y pretensiones para esta situación sean principios guía o ideales, estos están distorsionados. El caso de Luhmann es diferente; al prescindir de la situación ideal discursiva. No obstante el derecho tiene problemas sin resolver regados por todo el sistema; errores en su funcionamiento producidos por comunicaciones fallidas entre el sistema y las condiciones de cumplimiento de las reglas.

\section{CONCLUSIONES}

La racionalidad jurídica en la teoría Luhmanniana es producto de construcciones hechas al interior de un sistema de derecho. Esta construcción es el resultado del rompimiento con la teoría de la verdad como correspondencia, la influencia del lenguaje en los sistemas sociales, la autopoiesis en el sistema jurídico y el proceso de sustantivación objetivante. 
Los sistemas sociales como el derecho, en la teoría de Luhmann crean su racionalidad desde procesos autopoitéticamente cerrados; en estas construcciones cerradas el sistema crea la racionalidad del sistema a partir de comunicaciones que el mismo sistema crea o utiliza. La racionalidad en el derecho es una comunicación autopiética al interior del sistema. En esta comunicación es posible que sistemas afines al derecho coincidan-como el sistema moral o el económico-; pero en estas coincidencias no existe una relación necesaria de causalidad.

Las comunicaciones en el sistema de derecho son creadas en procesos de sustantivación objetivante; aquí se usa un lenguaje común, se agregan unas consecuencias determinadas por otras comunicaciones del derecho y de estas aparecen construcciones jurídicas; ejemplo de los procesos de sustantivación objetivamente pueden ser la teoría de los riesgos en el sistema de derecho civil, la teoría de la antijuricidad en el sistema de derecho penal o la teoría de la acción en el mismo sistema penal.

El derecho que explica Luhmann y Habermas, funciona a través de comunicaciones; reconocer esta afirmación implica ser conscientes de la posibilidad de que se emitan comunicaciones fallidas al interior del sistema.

\section{REFERENCIAS}

ALEXY, R. (2003) “Justicia como corrección”. En: Revista Doxa: cuadernos de filosofía del derecho, número 26. Universidad de Alicante.

APEL, K. (1991) "Teoría de la Verdad yética del discurso". Barcelona: Ediciones Paidós.

ELIAS; N. (2003) “Sobre el tiempo". México: Fondo de Cultura Económica.

FREUD, S. (2009) "Carta del Dr. Freud al profesor Einstein sobre la violencia y la guerra". En: Revista El Viejo Topo: №. 257, págs. 70-74.

GIRALDO ANGEL, J. (1985) "Metodología y técnica de la investigación jurídica". Bogotá: Librería del Profesional.

GÓMEZ-JARA DIEZ, C. (2008) "Teoría constructivista de la pena: ¿El dolor penal como constructo comunicativo?". En: Revista virtual Indret2 Abril: Revista para el análisis del derecho, Catalunya.

GUARDIOLA RIVERA, O. (2004) “El porvenir de una ilusión el futuro del derecho, desde la perspectiva de la teoría jurídica". En: Revista Vniversitas, diciembre, numero 108 pp. 711-732 Pontificia Universidad Javeriana

KAUFMANN, A (2002) “Filosofía del derecho”. Bogotá: Universidad Externado de Colombia.

KAUFMANN, A. (1992) "Panorámica histórica de los problemas de la filosofía del derecho". En: El pensamiento jurídico contemporáneo (95-120). Madrid: Debate. 
LUHMANN, N. ( 2005 ) "El derecho de la Sociedad". Recuperado en http://forodelderecho.blogcindario.com/2008/04/00432-el-derecho-de-la-sociedad-niklasluhmann.html

NEUMANN, U. (2006) "La Pretensión de verdad en el derecho y tres ensayos sobre Radbruch". Bogotá: Universidad Externado de Colombia.

TEUBNER, G. (2002) “El Derecho como sujeto epistémico: hacia una epistemología constructivista del derecho". En: Revista Doxa: cuadernos de filosofía del derecho, número 25. Universidad de Alicante. 